\title{
Calculation of Prestressed Pressure Vessel Taking into Account the Concrete Temperature Inhomogeneity
}

\author{
Vladimir Andreev ${ }^{1, *}$ \\ ${ }^{1}$ Moscow State University of Civil Engineering, Yaroslavskoe shosse, 26, Moscow, 129337, Russia
}

\begin{abstract}
The paper deals with the problem of determining the stress state of the pressure vessel $(\mathrm{PV})$ with considering the concrete temperature inhomogeneity. Such structures are widely used in heat power engineering, for example, in nuclear power engineering. The structures of such buildings are quite complex and a comprehensive analysis of the stress state in them can be carried out either by numerical or experimental methods. However, a number of fundamental questions can be solved on the basis of simplified models, in particular, studies of the effect on the stressed state of the inhomogeneity caused by the temperature field.
\end{abstract}

\section{Introduction}

In the PV which is usually constructed of reinforced concrete, under the influence of operational loads (internal pressure and temperature) there are very significant tensile stresses. In order to reduce or completely eliminate them, pre-compression in the annular and axial directions is used. Figure 1 shows a scheme of PV in which the compression in the circumferential direction is carried out by uniformly winding the metal cables along the outer surface, which leads to a constant pressure in the height [1]. Another way of pre-compression is to apply external pressure along the belts [2]. The corresponding scheme is shown in Fig.2.

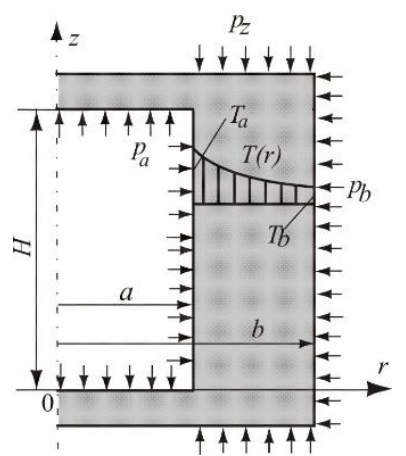

Fig. 1. Scheme pressure vessel.

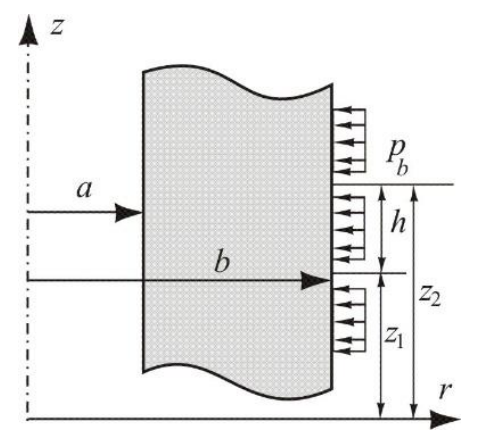

Fig. 2. Thick-walled cylinder with periodic loads. $h-$ load period.

\footnotetext{
*Corresponding author: asv@mgsu.ru
} 
Below are the results of calculating the stress state of the PV subjected to the action of a constant altitude temperature field $T(r)$, internal pressure $p_{a}$, pre-compression in the axial ( $p_{z}$ ) and circumferential $\left(p_{b}\right)$ directions. In this case, two methods of applying pressure $p_{b}$ - uniformly along the entire height of the PV and along the belts are considered.

\section{State of the problem}

We shall assume approximately that the change in temperature along the radius corresponds to the solution of the heat equation for a stationary temperature field in an infinitely long cylinder:

$$
T(r)=T_{b}+\left(T_{a}-T_{b}\right) \frac{\ln (b / r)}{\ln (b / a)}
$$

where $T_{a}$ and $T_{b}$ are respectively the temperatures on the inner and outer surfaces of the vessel. The dependence of the modulus of elasticity of concrete on temperature is given in accordance with [3] and approximated by a polynomial of the fourth degree. Fig. 3 shows the inhomogeneity functions $E(r)$ for several values $T_{a}$ at $T_{b}=20^{\circ} \mathrm{C}$. On the basis of the analysis performed in calculating forced (temperature) deformations $\varepsilon_{f}=\varepsilon_{T}$, the coefficient of linear temperature expansion in the temperature range under consideration varies insignificantly and we will assume it to be constant: $\alpha_{T}=\alpha_{T 0}$. Thus, the temperature deformations will be equal to $\varepsilon_{T}(r)=\alpha_{T 0} T(r)$.

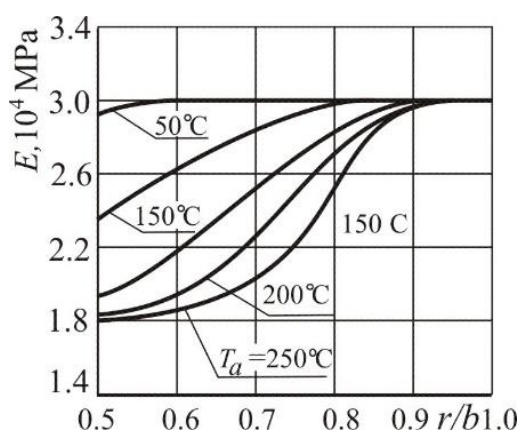

Fig. 3. Changing the modulus of elasticity in the wall of the PV.

\section{Method of solution}

The main task in this paper is the determination of the stress-strain state of the cylindrical part of the vessel. To calculate the thick-walled shell, with considering the inhomogeneity of the material, the numerical-analytical method described in $[4,5]$ was used. The solution of the axisymmetric problem is sought in displacements that are represented in the form of Fourier series:

$$
u(r, z)=u_{0}(r)+\sum_{n=1}^{\infty} u_{n}(r) \cdot \cos k_{n} z ; \quad w(r, z)=\sum_{n=1}^{\infty} w_{n}(r) \cdot \sin k_{n} z,
$$


where $k_{n}=n \pi / H$.

For a radially inhomogeneous body, the problem reduces to a system of ordinary differential equations of the second order [4]:

$$
\begin{aligned}
& (\lambda+2 \mu)\left(u_{n}^{\prime \prime}+\frac{u_{n}^{\prime}}{r}-\frac{u_{n}}{r^{2}}\right)-\mu k_{n}^{2} u_{n}+(\lambda+\mu) k_{n} w_{n}^{\prime}+\lambda^{\prime}\left[u_{n}^{\prime}+\frac{u_{n}}{r}+k_{n} w_{n}\right]+2 \mu^{\prime} u_{n}^{\prime}-3\left(K e_{n}\right)^{\prime}=0 \\
& \mu\left(w_{n}^{\prime \prime}+\frac{w_{n}^{\prime}}{r}-k_{n}^{2} w_{n}\right)-(\lambda+\mu) k_{n}\left(u_{n}^{\prime}+\frac{u_{n}}{r}+k_{n} w_{n}\right)+\mu^{\prime}\left(w_{n}^{\prime}-k_{n} u_{n}\right)+3 K k_{n} e_{n}=0 .
\end{aligned}
$$

Here, in the general case, $e_{n}$ are the expansion coefficients in the Fourier series of the temperature deformations $\varepsilon_{T}$.

Similarly, surface loads are decomposed into series. Since $\varepsilon_{T}$ and $e_{n}$ do not depend on $z$, they are represented by only one term of the Fourier series, and the corresponding coefficients and functions have the form:

$$
p_{a, 0}=2 p_{a} ; \quad p_{a, n}=0 ; \quad e_{0}=2 \varepsilon_{T}=2 \alpha_{0} T(r) ; \quad e_{n}=0(n=1,2 \ldots) .
$$

Pressure expansion coefficients for the case shown in Figure 2, is determined by formula

$$
p_{b, 0}=\frac{2 p_{b}\left(z_{2}-z_{1}\right)}{H_{1}} ; \quad p_{b, n}=\frac{2 p_{b}\left(z_{2}-z_{1}\right)}{n \pi H_{1}}\left[\sin \left(n \pi z_{2}\right)-\sin \left(n \pi z_{1}\right)\right] \text {. }
$$

Here $H$ - is the height of the layer corresponding to the load period. With uniform external lateral compression, $H$ can be chosen arbitrarily, for example, equal to unity, and $z_{1}=0, z_{2}=H$.

As a result of calculation determined displacement $u, v$, stresses $\sigma_{r}, \sigma_{\theta}$, and preliminary stresses $\sigma_{z}^{(1)}$ to which must be added the additional stresses $\sigma_{z}^{*}$ [6], defined by formula $\sigma_{z}^{*}=2 P E(r) / F^{*}$.

Here $F^{*}=2 \pi \int_{a}^{b} E(r) r d r-$ is the reduced area, and $P$ is the resultant force $P=P_{1}-P_{2}-P_{3}$ , where $P_{1}=p_{a} \pi a^{2}$ is the force resulting from the effect of pressure $p_{a}$ on the housing cover; $P_{2}=p_{z} \pi\left(b^{2}-a^{2}\right)$ - integral force from pre-compression in the axial direction; $P_{3}=2 \pi \int_{a}^{b} \sigma_{z}^{(1)} r d r-$ is the resultant of stresses $\sigma_{z}^{(1)}$ determined at the preliminary stage of the calculation.

The solution obtained with considering the stresses $\sigma_{z}^{*}$ is not exact in the whole region. In particular, the tangential stresses appearing in the contact zone of the PV wall with the cover, as well as the normal stresses caused by unbalanced bending moments from the stresses $\sigma_{z}^{(1)}$, remain unaccounted for. Thus, according to the principle of Saint-Venant, the results obtained will be valid in the middle part of the PV.

\section{Calculation of PV without considering the temperature inhomogeneity}


Fig. 4 shows the stress diagrams appearing in the PV wall from the operational loads $\left(p_{a}\right.$ and $T$ ) calculated with the following initial data: $a / b=0.5 ; \quad p_{a}=5 \mathrm{MPa} ; T_{a}=100^{\circ} \mathrm{C}$; $T_{b}=20^{\circ} \mathrm{C} ; \quad \alpha_{T 0}=0.12 \cdot 10^{-4} 1 /{ }^{\circ} \mathrm{C} ; E_{0}=3 \cdot 10^{4} \mathrm{MPa} ; v=0.2$. The diagrams $\sigma_{z}$ are constructed taking into account $\sigma_{z}^{*}$ without pressure $p_{z}$. The stresses $\sigma_{z}$ on the pressure $p_{z}$ are small, so that the total diagram of $\sigma_{z}$ is practically due only to the action of the temperature field. It can be noted that in the cylindrical part of the PV, very significant stresses arise $\sigma_{\theta}$ and $\sigma_{z}$ from the operational loads, which must be compensated by precompression pressures $p_{b}$ and $p_{z}$. First, it is necessary to choose the pressure $p_{b}$, since it leads to a change in the diagram of $\sigma_{z}^{(1)}$, then the pressure is selected, which does not affect the stresses $\sigma_{r}$ and $\sigma_{\theta}$.

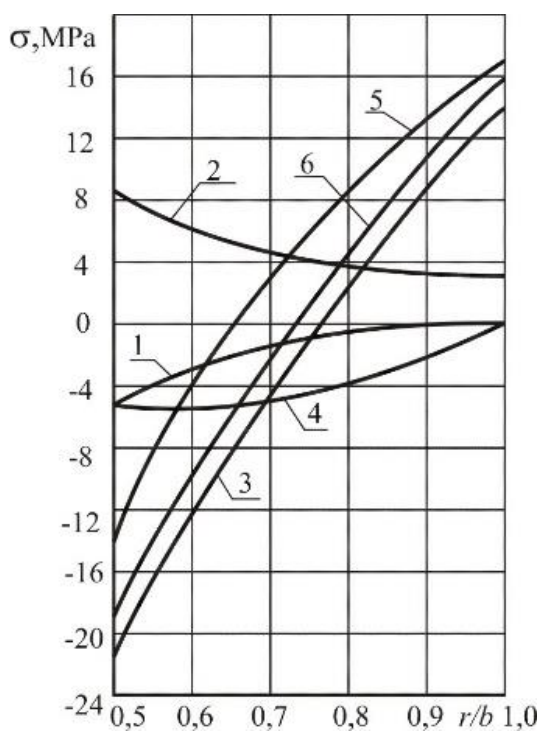

Fig. 4. Stresses in the wall of PV:

$1-\sigma_{r}\left(p_{a}\right) ; 2-\sigma_{\theta}\left(p_{a}\right) ; 3-\sigma_{z}\left(p_{a}\right) ;$

$4-\sigma_{r}\left(p_{a}, T\right) ; 5-\sigma_{\theta}\left(p_{a}, T\right) ; 6-\sigma_{z}\left(p_{a}, T\right)$.
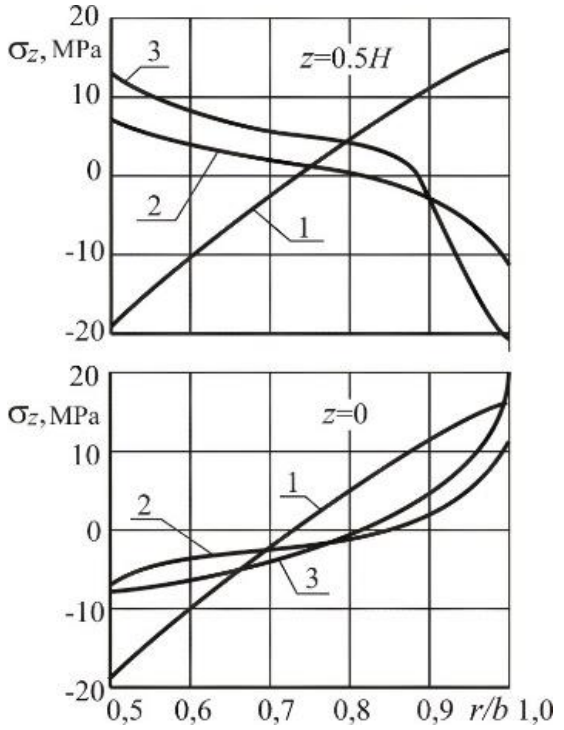

Fig. 5. Stresses in the wall of PV:

1 - from operational loads;

2, 3 - from external compression

$2-z_{1}=0.2 H ; \quad z_{2}=0.8 H ; 3-z_{1}=0.4 H ;$

$z_{2}=0.6 \mathrm{H}$.

Fig. 5 shows stress diagrams $\sigma_{z}$ at two levels $(z=0$ and $z=0.5 H)$ from operational loads ( $p_{a}=5 \mathrm{MPa} ; T_{a}=100^{\circ} \mathrm{C} ; T_{b}=20^{\circ} \mathrm{C} ;$ ) and for two cases of external compression $p_{b}$. In order for the integral value of the lateral compression to be constant the pressure value $p_{b}$ was chosen inversely proportional to the width of the load belt:

$$
p_{b}=\frac{p_{b}^{0} H}{z_{2}-z_{1}} .
$$

The pressure $p_{b}^{0}$ entering into this formula corresponds to a uniform compression over the entire outer surface. From the graphs shown in Fig. 5, it can be concluded that as the width of the belt decreases, the stresses $\sigma_{z}$ from pre-compression increase in absolute value 
both in the stretched and in the compressed zones. In this case, if in the section $z=0.5 H$ these stresses are compensated by stresses from the operational loads, then in the sections close to the end, these stresses are added together, and for the removal of tensile stresses with a narrower load belt, greater compressive forces in the axial direction are required.

\section{Calculation of PV with considering the temperature inhomogeneity of the material}

Stress state of PV is due to the common action of the internal pressure $p_{a}$, the temperature field and the pre-compression pressures $p_{b}$ and $p_{z}$. In order to visually observe the effect of temperature inhomogeneity on the stress state of the PV, the effect of each of these factors can be considered separately.

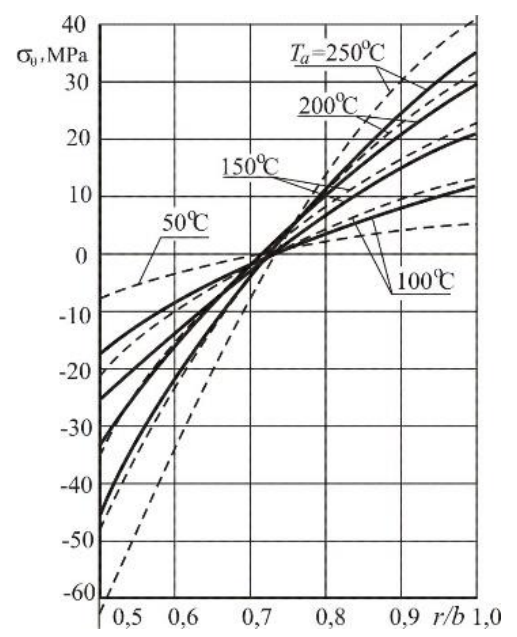

Fig.6. Stresses $\sigma_{\theta}$ in the wall of PV from the action of the temperature field:

— inhomogeneous material, - - - homogeneous material.

As an example, Fig. 6 shows the diagrams $\sigma_{\theta}$ in the wall of the PV from the action of the temperature field with considering and without considering the dependence $E(T)$ for different temperature values inside the shell (the temperature on the external surface is constant and equal $T_{b}=20^{\circ} \mathrm{C}$ ). The corresponding graphs $E(r)$ are shown in Fig. 3 . In the case when $T_{a}=50^{\circ} \mathrm{C}$, the stress values for homogeneous and inhomogeneous materials practically coincide. As the temperature rises, the modulus of elasticity decreases significantly, which leads to a decrease in stresses. These changes are more noticeable in the compressed zone, i.e. where the temperature is higher. A similar effect is observed when calculating the stresses from internal pressure $p_{a}$.

A noticeable decrease in stresses from operational loads, obtained with considering the temperature inhomogeneity of concrete, allows reducing the amount of pre-compression $p_{b}$, which in turn leads to a decrease in axial compression. For example, it is shown that for $T_{a}=200^{\circ} \mathrm{C}$ and for the width of the load belt $z_{2}-z_{1}=0.2 \mathrm{H}$, the pre-compression pressure $p_{b}$ with considering the inhomogeneity can be reduced by almost half compared to the calculation for a homogeneous material. 


\section{Conclusions}

Accounting for the inhomogeneity of materials introduces a significant correction to the stress-strain state of the structures. In the works [7, 8, etc.], the influence of various factors (radiation exposure, humidity, etc.) causing inhomogeneity of materials was considered. It is also possible to use this phenomenon for practical purposes. For example, $[9,10]$ describes a method for optimizing thick-walled concrete cylindrical shells by artificially changing the deformation properties of concrete.

This work was financially supported by the Ministry of Russian Education (state task \#7.1524.2017/PCh)

\section{References}

1. Altshuller M.A., Vinogradov V.N. and others, Nuclear power plants. 4, 17 (1981)

2. P.H. Margen, S. Mennon, J. of the British Nuclear Energy Soc. 14 1, 35 (1975)

3. Construction codes and standards 27.13330. (2011)

4. Andreev V.I. Applied Mechanics and Materials. 752-753 , 593 (2015)

5. Andreev V.I. Applied Mechanics and Materials. 752-753, 642 (2015)

6. Timoshenko S.P., Gudier Dj., Theory of elasticity (Nauka, Moscow, 1979)

7. Andreev V.I., Avershyev A. S., Int. J. for Comp. Civil and Struct Eng. 93 , 14 (2013)

8. Andreev V.I., Dubrovskiy I.A., Applied Mechanics and Materials. 405-408, 1073 (2013)

9. Andreev V.I., Applied Mechanics and Materials. 166-169, 354 (2012)

10. Andreev V.I., Potekhin I.A., Int. J. for Comp. Civil and Struct Eng.. 4 1, 79 (2008) 